\title{
Effectiveness of surgical left atrial appendage closure during minimally invasive mitral valve surgery
}

\author{
Grzegorz Hirnle, Janina Lewkowicz², Piotr Suwalski ${ }^{3}$, Maciej Mitrosz¹, Adam Łukasiewicz ${ }^{4}$, Tomasz Hirnle ${ }^{1}$ \\ 1 Department of Cardiac Surgery, Medical University of Bialystok, Białystok, Poland \\ 2 Department of Internal Medicine and Metabolic Diseases, Medical University of Bialystok, Białystok, Poland \\ 3 Clinical Department of Cardiac Surgery, Central Clinical Hospital of the Ministry of the Interior and Administration, Warsaw, Poland \\ 4 Department of Radiology, Medical University of Bialystok, Białystok, Poland
}

\section{KEY WORDS}

atrial fibrillation, left atrial appendage closure, minimally invasive mitral valve surgery

\section{Correspondence to:} Maciej Mitrosz, MD, PhD, Department of Cardiac Surgery, Medical University of Bialystok, ul. Marii Skłodowskiej-Curie 24A, 15-276 Białystok, Poland, phone: +48858318464 , email: mitrosz@gmail.com Received: April 17, 2020. Revision accepted: July 28, 2020. Published online: July 29, 2020. Kardiol Pol. 2020; 78 (11): 1137-1141 doi:10.33963/KP.15539 Copyright by the Author(s), 2020

\section{ABSTRACT}

BACKGROUND Left atrial appendage closure (LAAC) reduces the risk of stroke in patients with atrial fibrillation. It can be performed surgically from the inside of the left atrium or from the outside. Stapling or clipping devices can also be used from the outside. Despite providing an excellent interior view of the appendage, those techniques cannot be implemented during minimally invasive mitral valve surgery conducted through right-sided minithoracotomy.

AIms This study aimed to assess the effectiveness of surgical closure of the left atrial appendage from the inside during minimally invasive mitral valve surgery.

METHODS A total of 50 patients with mitral valve disease and atrial fibrillation who underwent minimally invasive mitral valve surgery and LAAC between 2012 and 2017 were included in this study. The appendage was closed from the inside using a continuous suture. After a median follow-up of 1.6 years after surgery, 19 patients were examined by transthoracic and transesophageal echocardiography (TEE). Transesophageal echocardiography was performed to assess whether the appendage had been effectively closed. When any leakage was suspected, cardiac computed tomography was performed.

RESULTS In 19 patients, TEE was performed at 0.5 to 5 years after the surgery. A single patient did not tolerate TEE, and minimal leakage was suspected in 2 patients. All 3 individuals underwent computed tomography examination, which confirmed leakage in a single patient.

CONCLUSIONS Surgical LAAC during minimally invasive mitral valve surgery through right minithoracotomy is an effective technique that provides durable results.

INTRODUCTION Ischemic stroke is a serious complication of atrial fibrillation (AF). It has been reported that more than $90 \%$ of thrombi responsible for cerebral ischemia originate from the left atrial appendage (LAA). ${ }^{1}$ For this reason, left atrial appendage closure (LAAC) was introduced as a method of thromboprophylaxis. Due to the insufficient effectiveness of antiarrhythmic medications, anticoagulants remain the mainstay of therapy for patients with AF. Unfortunately, these drugs may significantly increase the bleeding risk and impair the quality of life. ${ }^{2}$ Therefore, in patients with AF, LAAC is an alternative both as a concomitant and an isolated procedure. ${ }^{3-5}$

Appendage closure can be performed surgically by suturing it either from the inside of the left atrium or from the outside. It is usually combined with appendage amputation. Among classic surgical approaches, only the "from the outside" method combined with appendage amputation yields durable results. However, the procedural success is achieved only if the residual stump does not exceed 


\section{WHAT'S NEW?}

Left atrial appendage occlusion decreases the risk of stroke complications in patients with atrial fibrillation. Surgical closure of the left atrial appendage during full sternotomy is a common procedure, whereas data on surgical closure during minimally invasive mitral valve surgery have not been reported so far. This study shows the satisfactory effectiveness of the latter approach performed through right minithoracotomy using the double-row suturing method. Minimally invasive mitral valve surgery provides very good visibility of the base of the atrial appendage and thus facilitates complete suturing. As minimally invasive methods has become common in cardiac surgery, the described method of atrial appendage occlusion may be expected to gain even greater importance.

$1 \mathrm{~cm} .{ }^{6}$ Secure closure from the outside is also possible with clips or cutting staplers, which could be applied both during operations via sternotomy as well as left minithoracotomy or even during thoracoscopic surgeries with access through the left pleural space. Percutaneous devices have been introduced to exclude the left atrial appendage from the inside and thereby eliminate the risk of blood retention and clot formation. Appendage exclusion performed with percutaneous devices has provided good results in selected patients with favorable anatomy. Furthermore, leaks around the device not exceeding $5 \mathrm{~mm}$ are regarded as a procedural success. However, even if such leakage occurs, a significant decrease in the stroke rate is reported. ${ }^{7}$

So far, no studies have examined the effectiveness of surgical appendage closure as a concomitant procedure performed during minimally invasive mitral valve surgery. Therefore, the aim of our study was to assess the effectiveness of surgical closure of the LAA during minimally invasive mitral valve surgery via right minithoracotomy.

METHODS Study population We conducted a retrospective study and analyzed patients' archived anonymous data. In the years from 2012 to 2017, 50 consecutive patients with mitral valve disease underwent minimally invasive mitral valve surgery and LAAC. All of them were invited to echocardiographic examination in order to assess the outcomes of LAAC. A total of 19 patients responded to the invitation and were included in the study. The baseline clinical characteristics of the study patients are presented in TABLES 1 and 2.

Surgery The surgeries were performed by an experienced cardiovascular surgeon. In all study patients, the access site was achieved by right minithoracotomy in the 4 th intercostal space. Extracorporeal circulation was established through the groin vessels.
The appendage was sutured from the inside using a double-row monofilament 3-0 continuous suture. The first row was placed on the transition of the atrial and appendage walls. The second row invaginated the first suture line outside from the atrium. This method eliminates the possibility of leaving any residual stump inside.

Imaging of the left atrium After minimally invasive mitral valve surgery and LAAC, all 19 patients underwent complete 2-dimensional transthoracic echocardiography (TTE) and transesophageal echocardiography (TEE) with color Doppler imaging of the LAA.

Conventional 2-dimensional TTE and TEE were performed by the same experienced echocardiographer, using commercially available transducers and equipment (the S5-1 probe for TTE and the X7-2t probe for TEE; Philips CX 50, Philips Healthcare, Andover, Massachusetts, United States), and in the same echocardiography laboratory. In each study participant, cardiac anatomy and function were comprehensively assessed by ultrasonography.

Standard TEE was performed in all study patients (particularly focused on evaluating the LAA). The LAA was examined in multiple views with the use of color Doppler imaging to assess the presence of blood flow between the left atrium and the closed appendage. Furthermore, 3-dimensional imaging was used to evaluate the structure and related anatomy.

Cardiac computed tomography (CT) was performed when any leakage was suspected.

Computed tomography angiography was conducted using the Aquilion One 320-row detector (Toshiba Medical Systems, Tokyo, Japan). Nonionic contrast material $(60 \mathrm{ml})$, iomeprol (Iomeron 400, Bracco Imaging SpA, Milan, Italy), followed by $30-\mathrm{ml}$ solution ( $50 \%$ contrast $/ 50 \%$ saline) was administered intravenously at a rate of $5 \mathrm{ml} / \mathrm{s}$ using a power injector. Cardiac CT protocols were obtained using 2-phase scanning.

Digital images were interpreted with the VitreaWorkstation software (Vital, Toshiba Medical Systems Group Company, Veenendaal, The Netherlands).

The university ethics committee approved the study (APK.002.213.2020), and patient written consent for examination was obtained.

Statistical analysis Due to an extremely small sample size (only a single patient with incomplete occlusion) and low statistical power, comparisons between complete and incomplete appendage occlusions were omitted. Study results were expressed with descriptive statistics. The IBM SPSS Statistics software, version 20.0 (Armonk, New York, United States), was used for calculations. 
TABLE 1 Baseline characteristics of the study patients

\begin{tabular}{lll} 
Characteristics & Mean (SD) & Median (IQR) \\
Age, y & $67.4(10.3)$ & $70(62-75)$ \\
\hline LA size, cm & $5(0.6)$ & $5.1(4.4-5.6)$ \\
\hline LA volume, $\mathrm{ml}$ & $128.2(44.9)$ & $133(79-160)$ \\
\hline LAV index, $\mathrm{ml} / \mathrm{m}^{2}$ & $68.4(24.2)$ & $70(46-86)$ \\
\hline EF, \% & $48.5(10)$ & $52(40-55)$ \\
\hline NT-proBNP, pg/ml & $524.5(463.2)$ & $401.8(221.9-724.8)$ \\
\hline Creatinine, $\mathrm{mg} / \mathrm{dl}$ & $1(0.3)$ & $0.9(0.8-1.1)$ \\
\hline eGFR, ml/min/1.73 m² & $70.3(21.1)$ & $69(53-79)$ \\
\hline Total cholesterol, mg/dl & $176.9(78.5)$ & $170(112.8-213.8)$ \\
\hline LDL cholesterol, mg/dl & $119.8(53.8)$ & $120.5(78-144.3)$ \\
\hline HDL cholesterol, mg/dl & $43.9(14.7)$ & $43.5(36.8-56.5)$ \\
\hline Triglycerides, mg/dl & $100.5(134.4)$ & $78(0-117)$ \\
\hline Hemoglobin, mg/dl & $13.27(1.49)$ & $13.1(12.4-13.9)$ \\
\hline
\end{tabular}

Abbreviations: EF, ejection fraction; eGFR, estimated glomerular filtration rate; HDL, high-density lipoprotein; LA, left atrium; LAV, left atrium volume; LDL, low-density lipoprotein; NT-proBNP, N-terminal fragment of the prohormone brain natriuretic peptide

TABLE 2 Comorbidities of the study patients

\begin{tabular}{lll} 
Comorbidity & & Patients, $\mathbf{n}(\%)$ \\
Hypertension & $12(63.2)$ \\
\hline History of stroke & $2(10.5)$ \\
\hline Heart failure & NYHA class I & $1(5.3)$ \\
\cline { 2 - 3 } & NYHA class II & $12(63.2)$ \\
\cline { 2 - 3 } & NYHA class III & $6(31.5)$ \\
\hline
\end{tabular}

Abbreviations: NYHA, New York Heart Association

RESULTS Among the study patients, there were 12 women aged between 61 to 73 years and 7 men aged between 36 to 76 years. The mean (SD) age of the operated patients was 67.4 (10.3) years. All of them underwent intraoperative TEE performed by an anesthesiologist, which demonstrated a good result of the appendage closure immediately after the surgery.

Time elapsed from surgery to TEE examination ranged from 0.5 to 5 years. No study patient suffered from stroke or other thromboembolic events during follow-up. In a single patient, TEE was not performed because of poor toleration of the TEE probe. Out of 19 patients, complete occlusion was confirmed in 18 individuals (95\%). During TEE examination, a trace leakage not exceeding $1 \mathrm{~mm}$ was suspected (FIGURE 1 ) in 2 patients. In both cases, the suspicion of leakage was ruled out on CT (FIGURE 2). In a single patient, in whom TEE was not performed, CT showed a significant leakage of the LAA suture line of about $6 \mathrm{~mm}$ in diameter. Selected procedural and imaging data are presented in TABLE3.
DISCUSSION An effective occlusion of the LAA remains a challenge regardless of the closure technique used. In the largest transcatheter closure registry, EWOLUTION (Evaluating Real-Life Clinical Outcomes in Atrial Fibrillation Patients Receiving the WATCHMAN Left Atrial Appendage Closure Technology), the device did not expand in 15 individuals out of 1019 patients admitted for appendage closure with the Watchman system, left a leak less than $5 \mathrm{~mm}$ in 78, and caused a leak larger than $5 \mathrm{~mm}$ in 7. A total of 100 patients (9.8\%) did not achieve optimal procedural outcomes. ${ }^{7}$ According to the liberal criteria set in that registry, only a leakage above $5 \mathrm{~mm}$ was regarded as a procedural failure. On the contrary, in surgical records, only complete appendage occlusion without a residual stump is considered a procedural success. According to these criteria, the LAAOS (Left Atrial Appendage Occlusion Study) showed procedural success in surgical closure only in $45 \%$ of patients. Interestingly, after the operator had performed at least 


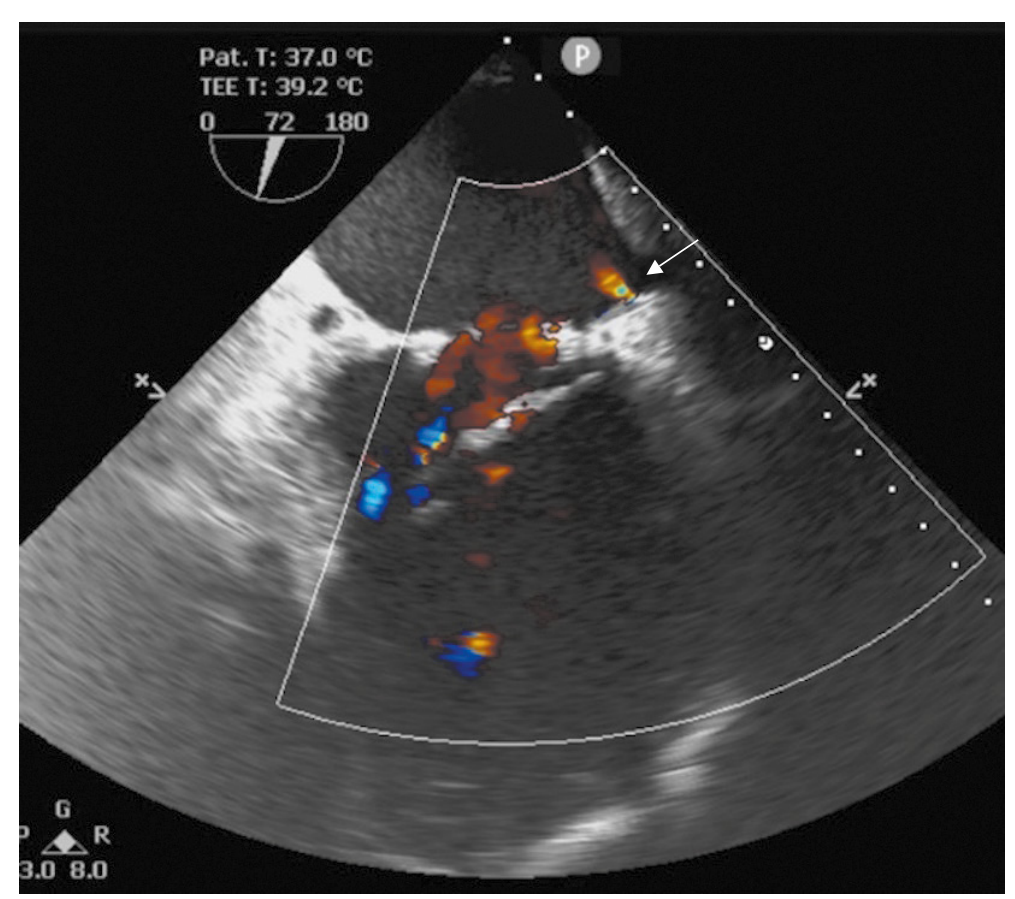

FIGURE 1 Suspected residual flow and persistent leakage after surgical left atrial appendage closure on transesophageal echocardiography (arrow)

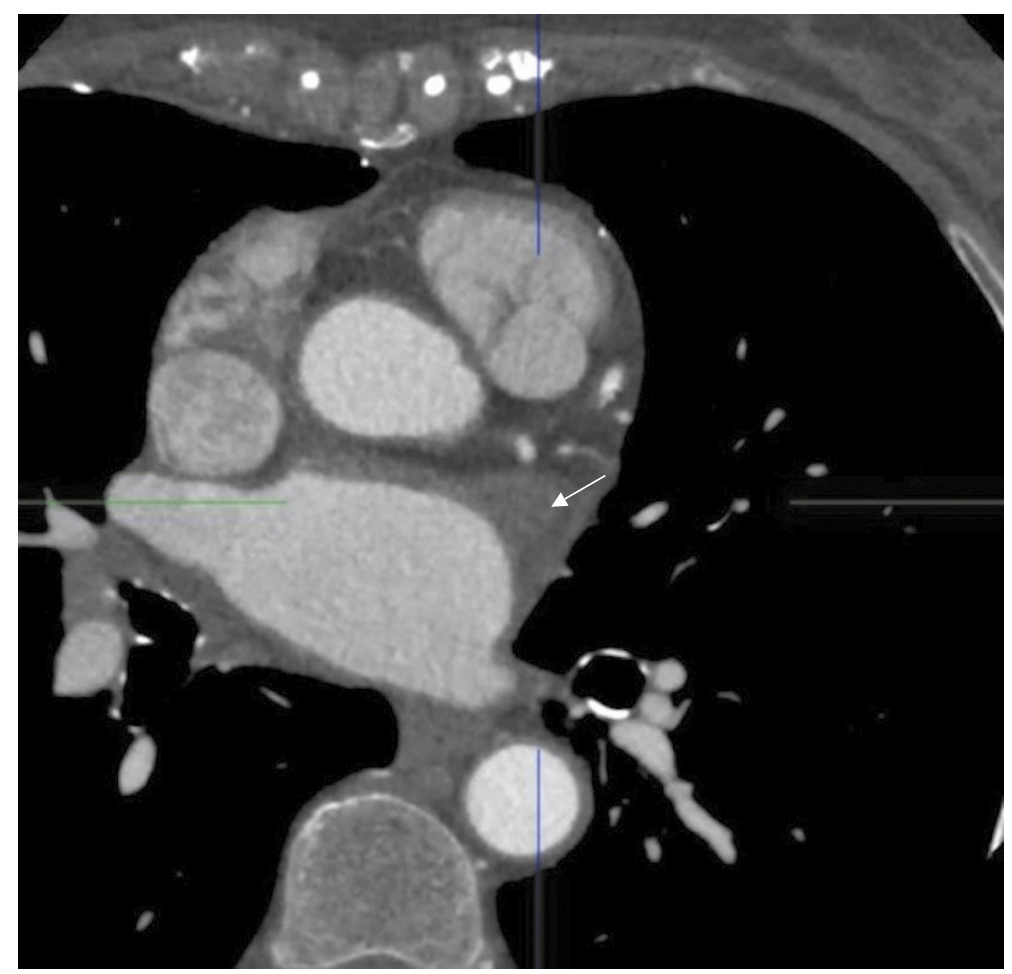

FIGURE 2 Computed tomography showing a complete occlusion of the appendage: a stump of the left atrial appendage without blood flow (arrow)

4 procedures, the procedural success doubled to $87 \% .^{8}$ Therefore, the credibility of this observation seems to be questionable. Despite low-grade guideline recommendations, surgical LAAC is intuitively performed in patients with AF undergoing concomitant procedures. A recent meta-analysis including 280585 patients demonstrated that LAAC reduces the incidence of postoperative stroke or embolism and mortality in patients with preoperative AF. ${ }^{4}$

Based on the literature, it can be assumed that anticoagulation is not necessary in patients with an occluded appendage. ${ }^{9}$ It seems that, after confirming the successful surgical closure of the LAA on TEE or CT, discontinuation of anticoagulation can be considered.

Minimally invasive mitral valve surgery provides very good visibility of the atrial appendage, which facilitates complete suturing. We achieved complete closure in $95 \%$ of the study patients. Thus, the effectiveness of our method at mid-term follow-up was definitely greater than that of the currently used transcatheter closure technique (95\% vs $44 \%-48 \%)$. $^{10,11}$ Furthermore, suturing with a double-row technique from the inside eliminates the risk of leaving a residual stump inside. Of note, in contrast to the transcatheter closure technique, every anatomical variation of the appendage is suitable for surgical closure.

The number of mitral valve surgeries performed with minimally invasive access is constantly rising. Therefore, an effective method of LAAC during mitral valve surgery with right-sided minithoracotomy may have a prognostic value for larger numbers of patients. To confirm these data, further evaluation and multicenter studies are needed.

There are 2 modalities widely used to assess the effectiveness of the LAAC procedure: TEE and CT. Computed tomography is a noninvasive method. Moreover, it has excellent spatial resolution, and 3-dimensional assessment facilitates more sensitive detection and clarifies the mechanisms of leakage. The disadvantages of CT scanning include the need for using a contrast agent, radiation exposure, high costs, and still limited availability. In our study, none of the cases of incomplete LAAC detected by TEE were confirmed by CT. This is in contrast to previous reports, ${ }^{10,11}$ in which left atrial appendage patency was observed more often on CT. Overdiagnosis of leakage on TEE with color Doppler imaging may result from the incorrect assessment of blood flow in the circumflex artery.

Study limitations Admittedly, our study included a small number of patients. Its results might be underpowered to determine the true clinical benefits of the presented technique of surgical LAAC.

Conclusions Surgical suturing of the left atrial appendage as a concomitant procedure during minimally invasive mitral valve surgery performed through right minithoracotomy is an effective technique that provides durable results. In patients without kidney disease, $C T$ is a valuable alternative to TEE in the assessment of LAAC efficacy. 
TABLE 3 Procedural and imaging data of the study patients

\begin{tabular}{|c|c|c|c|c|c|c|c|}
\hline Patient no. & Age, $y$ & Sex & Procedure & $\begin{array}{l}\text { Time elapsed from } \\
\text { surgery to TEE, } y\end{array}$ & $\begin{array}{l}\text { LAAC outcome } \\
\text { on TEE }\end{array}$ & $\begin{array}{l}\text { LAAC outcome } \\
\text { on CT }\end{array}$ & $\begin{array}{l}\text { Residual } \\
\text { stump }>1 \mathrm{~cm} \text { on TEE }\end{array}$ \\
\hline 1 & 72 & Female & MVR & 5 & Complete & - & No \\
\hline 2 & 66 & Female & MVR, TVR & 5 & Complete & - & No \\
\hline 3 & 70 & Male & $\begin{array}{l}\text { Mitral valve repair, } \\
\text { TVR, ablation }\end{array}$ & 2 & Complete & - & No \\
\hline 4 & 65 & Male & MVR & 1.5 & Complete & - & No \\
\hline 5 & 73 & Female & $\begin{array}{l}\text { Mitral valve repair, } \\
\text { TVR }\end{array}$ & 1.6 & Complete & - & No \\
\hline 6 & 61 & Female & MVR & 1.6 & Complete & - & No \\
\hline 7 & 36 & Male & $\begin{array}{l}\text { Mitral valve repair, } \\
\text { TVR }\end{array}$ & 1.4 & Not performed & Incomplete & - \\
\hline 8 & 67 & Male & $\begin{array}{l}\text { Mitral valve repair, } \\
\text { TVR }\end{array}$ & 2.1 & $\begin{array}{l}\text { Trace leakage } \\
(<1 \mathrm{~mm})\end{array}$ & Complete & No \\
\hline 9 & 63 & Female & MVR, TVR, ablation & 2 & Complete & - & No \\
\hline 10 & 70 & Female & $\begin{array}{l}\text { Mitral valve repair, } \\
\text { TVR }\end{array}$ & 1.6 & Complete & - & No \\
\hline 11 & 76 & Female & $\begin{array}{l}\text { Mitral valve repair, } \\
\text { TVR, PFO closure }\end{array}$ & 1.5 & $\begin{array}{l}\text { Trace leakage } \\
(<1 \mathrm{~mm})\end{array}$ & Complete & No \\
\hline 12 & 65 & Female & MVR, TVR, ablation & 0.5 & Complete & - & No \\
\hline 13 & 72 & Male & $\begin{array}{l}\text { Mitral valve repair, } \\
\text { TVR }\end{array}$ & 0.5 & Complete & - & No \\
\hline 14 & 59 & Female & $\begin{array}{l}\text { Mitral valve repair, } \\
\text { TVR, ablation }\end{array}$ & 2.8 & Complete & - & No \\
\hline 15 & 76 & Male & $\begin{array}{l}\text { Mitral valve repair, } \\
\text { TVR }\end{array}$ & 1.8 & Complete & - & No \\
\hline 16 & 76 & Male & Mitral valve repair & 1.8 & Complete & - & No \\
\hline 17 & 55 & Female & $\begin{array}{l}\text { Mitral valve repair, } \\
\text { TVR, ablation }\end{array}$ & 4.3 & Complete & - & No \\
\hline 18 & 53 & Female & MVR, TVR, ablation & 1 & Complete & - & No \\
\hline 19 & 57 & Female & MVR, TVR, ablation & 1 & Complete & - & No \\
\hline
\end{tabular}

Abbreviations: CT, computed tomography; LAAC, left atrial appendage closure; MVR, mitral valve replacement; PFO, patent foramen ovale; TEE, transesophageal echocardiography; TVR, tricuspid valve repair

\section{ARTICLE INFORMATION}

\section{CONFLICT OF INTEREST None declared.}

OPEN ACCESS This is an Open Access article distributed under the terms of the Creative Commons Attribution-Non Commercial-No Derivatives $4.0 \mathrm{In}-$ ternational License (CC BY-NC-ND 4.0), allowing third parties to download articles and share them with others, provided the original work is properly cited, not changed in any way, distributed under the same license, and used for noncommercial purposes only. For commercial use, please contact the journal office at kardiologiapolska@ptkardio.pl.

HOW TO CITE Hirnle G, LewkowiczJ, SuwalskiP, et al. Effectiveness of surgical left atrial appendage closure during minimally invasive mitral valve surgery. Kardiol Pol. 2020; 78: 1137-1141. doi:10.33963/KP.15539

\section{REFERENCES}

1 Hanke T. Surgical management of the left atrial appendage: a must or a myth? Eur J Cardiothorac Surg. 2018; 53 (suppl 1): i33-i38.

2 Romano MA, Bergquist CS. Left atrial appendage management: $n$ o better time than the present. Innovations. 2019; 14: 498-502.

3 Ismail TF, Panikker $S$, Markides $\mathrm{V}$, et al. CT imaging for left atrial appendage closure: a review and pictorial essay. J Cardiovasc Comput Tomogr. 2015; 9: 89-102.

4 Martín Gutiérrez E, Castaño M, Gualis ], et al. Beneficial effect of left atrial appendage closure during cardiac surgery: a meta-analysis of 280585 patients. Eur J Cardiothorac Surg. 2020; 57: 252-262.
5 Tsai YC, Phan K, Munkholm-Larsen S, et al. Surgical left atrial appendage occlusion during cardiac surgery for patients with atrial fibrillation: a meta-analysis. Eur J Cardiothorac Surg. 2015; 47: 847-854.

6 Squiers JJ, Edgerton JR. Surgical closure of the left atrial appendage: the past, the present, the future. J Atr Fibrillation. 2018; 10: 1642.

7 Boersma LV, Ince H, Kische S, et al. Efficacy and safety of left atrial appendage closure with WATCHMAN in patients with or without contraindication to oral anticoagulation: 1-Year follow-up outcome data of the EWOLUTION trial. Heart Rhythm. 2017; 14: 1302-1308.

8 Healey J, Crystal E, Lamy A, et al. Left Atrial Appendage Occlusion Study (LAAOS): results of a randomized controlled pilot study of left atrial appendage occlusion during coronary bypass surgery in patients at risk for stroke. Am Heart J 2005; 150: 288-293.

9 Grygier M, Wojakowski W, Smolka G, et al. Left atrial appendage occlusion: consensus document of Association of Cardiovascular Interventions and Heart Rhythm Section of Polish Cardiac Society [in Polish]. Kardiol Pol. 2018; 76, 3: 677-697.

10 Qamar SR, Jalal S, Nicolaou S, et al. Comparison of cardiac computed tomography angiography and transoesophageal echocardiography for device surveillance after left atrial appendage closure. EuroIntervention. 2019; 15: 663-670. 11 Cochet $H$, Iriart $X$, Sridi $S$, et al. Left atrial appendage patency and device-related thrombus after percutaneous left atrial appendage occlusion: a computed tomography study. Eur Heart J Cardiovasc Imaging. 2018; 19: 1351-1361. 